\title{
Modification of the Basal View after Internal Alar Reduction
}

\author{
LAILA A. ABOUL NASR, M.D.; SARAH S. RAAFAT, M.Sc.; MOTAZ NATSHA, M.Sc.; \\ SHERIF Z. EZZAT, M.D. and YOUSSEF KHACHABA, M.D.
}

The Department of Plastic Surgery, Faculty of Medicine, Cairo University, Egypt

\begin{abstract}
Background: Numerous alar base modification techniques have been reported in the literature. Specifically, three different techniques were described to manage cases with alar flaring in addition to a wide nasal base. Tissue excision from the alar lobule as well as from the inside of the nostril is executed to decrease the alar flaring and the nostril width, respectively. Further techniques include cinching sutures that help advance the alae together and flap advancement.
\end{abstract}

The aim of this study is to emphasize the aesthetic impact of the basal nasal view and to reach a maximum aesthetic result concerning nasal alar flare.

Patients and Methods: A prospective study was conducted on 25 patients. All patients had alar flaring seeking alar reduction, either alone or as a part of a full rhinoplasty. During rhinoplasty, alar base reduction was done at the end of rhinoplasty surgery, after the closure of all rhinoplasty incisions and after osteotomy was done. A caliper was used to sharply stab the skin from the lateral limb to the desired sill crease to determine the medial limb of the triangular wedge to be excised. The base of the triangular wedge was directed downward and the apex was directed intra-nasally.

Results: The current study showed that the internal alar resection significantly decrease the alar flare; as the inter-alar distance ranged between (35-43mm), Mean \pm SD (39.2 \pm 2.3 ) before surgery and becomes between (31-37mm) Mean \pm SD $(34.8 \pm 2.0)$ after the surgery, with a significant improvement ( $p$-value: $<0.001)$.

Conclusion: Internal alar resection effectively reduced the alar flare, and improved the nostril shape and width. This technique was also useful to retain symmetry of nostril shape, hide the scars internally in the nose, avoid the injury of the lateral nasal artery, preserve the natural curvature of alar rim and avoid detachment of the alar foot plate. This technique can effectually be applied to all patients with alar flare reduction.

Key Words: Rhinoplasty - Alar reduction - Internal - Basal view.

\section{INTRODUCTION}

The preoperative evaluation of nasal soft tissues is a fundamental part of rhinoplasty surgery success. It is of utmost importance to achieve an esthetic balance and natural appearance when modifying the soft tissues of the nasal tip, alae and nostrils [1].
The nose should appear aesthetically balanced to the rest of the face [2]. The width of the nose should relate to the concept of vertical fifths of face. The alae should lie between both medial canthi and should be equal to the distance between medial and lateral canthus [3].

Alar flaring and nostril size may be associated with diverse ethnicity, history of trauma, history of cocaine abuse or secondary rhinoplasty $[\mathbf{1 8 , 2 0 ]}$.

The concept of narrowing the alar base dates back to the early days of modern rhinoplasty when Robert Wier in 1892, described the use of external alar wedge excisions to correct the unattractive alar flare that follows rhinoplasty [4].

Joseph and Milstein used internal excisions in 1931 from the nostril base and floor of the vestibule [4]. This concept was later modified and popularized by many authors, mainly to avoid the external scar that follows the classic Wier excision [5].

Numerous alar base modification techniques have been reported in literature. Specifically, three different techniques were described to manage cases with alar flaring in addition to a wide nasal base. Tissue excision from the alar lobule as well as from the inside of the nostril is executed to decrease the alar flaring and the nostril width, respectively. Further techniques include cinching sutures that help advance the alae together and flap advancement [6]. Cinching sutures can be used in cases with minimal flaring with the advantage of avoiding scars. To achieve better results some authors modified the technique and combined it with excisions from vestibular floor or alar lobule [7]. In 2014 Hamilton described the Y-to-V advancement flap, in which there is no tissue removal rendering it adjustable and reversible [8].

The dimensions of the alar base width which are measured from one alar crease to the opposite ought to ideally be equivalent to the intercanthal distance [3]. 
The maximum point of alar convexity beyond the alar crease is defined as the alar flare. This distance should ideally be within two millimeters. When a line is drawn from the medial canthi downwards, the alae should not extend beyond it [9].

Aristotle the Greek philosopher had described beauty as "an imprecise sense of harmonious or aesthetically pleasing proportionality". The golden ratio can be used when describing beauty and symmetry [10]. Symmetry is one of the desired aesthetic goals and is a determining factor in rhinoplasty satisfaction. Achieving symmetry is difficult and can be affected by nasal tip asymmetry, caudal septum deviation, base asymmetry and flaring variations [6].

The surgical step of internal alar reduction is performed as the last step of rhinoplasty surgery, or can be performed alone if alar flare is the only complaint [6]. In this technique, the amount of the lobule to be resected is planned and marked. The incision is made from the nasal sill side. A small triangular wedge is then excised from the medial wall of alar base. It is important to preserve the natural alar-cheek junction with this incision and not to extend to the alar crease in order to avoid injury of the lateral nasal artery [6] (Figs. 1,2).

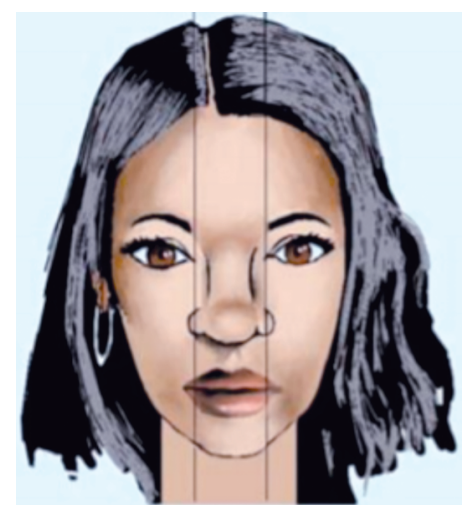

Fig. (1): Alar flare Lima et al., 2016.

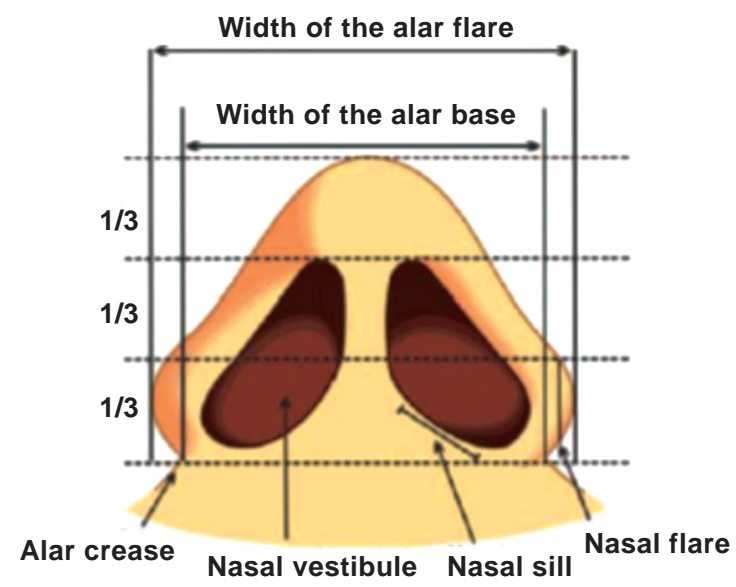

Fig. (2): Nasal base anatomy Lima et al., 2016.
Internal alar reduction has many advantages including abscence of external scar and preservation of the natural curvature of alar rim [4].

The alar anatomy is unique and of great importance; as it not only affects the basal view morphology, but also its dynamic relationship with the tip affects projection and flare [12].

The basal nasal view is significant when evaluating the nasal tip contour and alae. It is important to check this view to assess the relationship between the nasal tip and alar lobule and to have a natural smooth transition between them. The "ideal tiplobule line" is the line described by Casanueva and Gerecci connecting the tip-defining point to alar lobule on each side [11].

Attaining patient satisfaction by decreasing nasal flare without scars, and with maximum safety is the main goal. The method presented in this study is a simplified approach that avoids injury of lateral nasal artery, avoids the detachment of alar foot plate, and avoids nasal ala deformity.

The aim of this study is to emphasize the aesthetic impact of the basal nasal view and to reach maximum aesthetic results concerning nasal alar flare.

\section{PATIENTS AND METHODS}

Study design:

This study was conducted at Kasr Al-Aini University Hospital, Department of Plastic and Reconstructive Surgery, during the period from March 2019 till January 2020.

This study was accepted by the scientific and ethical committee of plastic surgery department of Cairo University hospitals. Patients provided verbal and written consent for the procedure and participation in the study.

This prospective study included 25 patients, 19 females and 6 males, between the age of 20 and 37 years. All patients had alar flaring in constitutional non-traumatic non-congenital nose, and seeking alar reduction, either alone or as a part of full rhinoplasty. Both primary and secondary rhinoplasty patients were included in this study. Reconstructive cases were excluded as well as patients with medical comorbidities, such as diabetes mellitus, hypertension, bleeding tendency or local nasal pathology such as rhinophyma and acne.

\section{Preoperative preparation:}

A detailed history was obtained from patients, including personal data, previous surgeries, and 
drug allergies. Full functional and aesthetic nasal analysis was performed during the examination as part of the typical rhinoplasty preoperative preparation.

From the frontal view facial proportions, skin type and quality, symmetry and nasal deviation, bony vault, mid vault, dorsal aesthetic lines, nasal tip, alar rims, alar base and upper lip were assessed. Lateral view analysis included naso-frontal angle, nasal length, dorsum, supratip break, tip projection, tip rotation, alar-columellar relationship, periapical hypoplasia and lip-chin relationship. Dorsum deviation was checked from the top view.

In our cases, where basal morphology was found different, special attention was made to the basal view to check nasal projection, caudal septal deviation, columella, alar base, alar flaring and nostrils.

Measurements included intercanthal distance: (n: 30-34 mm), alar base (measured from one alar crease to the other), alar flare: Maximum degree of alar convexity beyond the alar crease, columellar width, nostrils width, alar width, tip and columellar length.

Photography was performed on the patients preoperatively, intraoperative, and postoperatively. Pre and postoperative views included frontal, basal, lateral, and oblique views.

X-rays were only done in secondary and posttraumatic cases to assess the degree of deformity.

\section{Consent:}

Before the surgery, potential complications were explained to all patients and a signed standard informed consent was obtained.

\section{Operative technique:}

Surgery was performed with patients in the supine position. In full rhinoplasty cases, the surgery was performed under general anesthesia. While in cases of alar reduction alone, the procedure was done using local anesthesia.

The face and nose were prepped and draped using standard aseptic technique. Cotton-tipped applicators were used to clean the nasal vestibules.

Alar base narrowing was performed as the final step in rhinoplasty, after closure of all rhinoplasty incisions and after osteotomy was done, so the amount of alar base narrowing could be judged properly. The mid-columellar point was marked first. A ruler was then used to measure the distance from the mid-columellar point to the alar crease, on each side, to detect any difference in width of the nasal sill which may require asymmetric excisions from the nasal sills. Compression on the lateral wall of the ala is of importance to determine the lateral limb of the triangular wedge to be excised. A caliper was used to detect any asymmetry in the marking of the lateral limb of the triangular wedge. The desired amount of narrowing of the outer diameter of the ala was determined. Caliper was used to sharply stab the skin from the lateral limb to desired sill crease to determine the medial limb of the triangular wedge to be excised. The base of the triangular wedge was directed downward and the apex was directed intra-nasally. (Fig. 3).

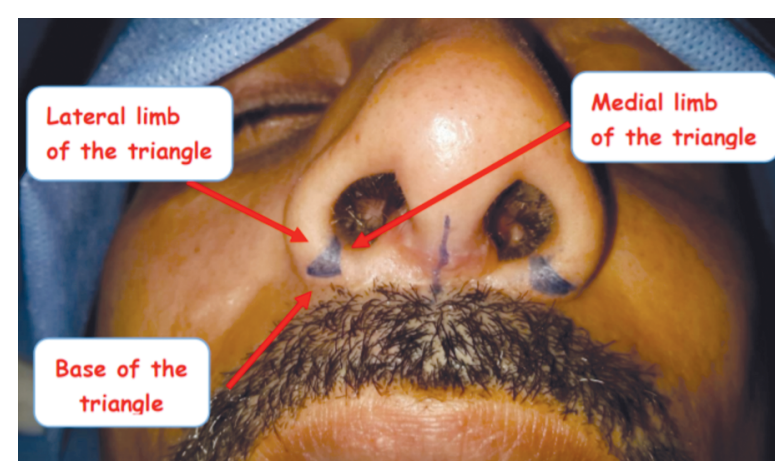

Fig. (3): Triangular wedge markings.

After injecting the alar base with a solution (2\% lidocaine and 1:100,000 epinephrine), 15 minutes were allowed for vasoconstriction to take place. Lateral and then medial sill incisions were made with a number 15 blade and the triangular wedge of skin was removed, ensuring that the deep muscular layer remains intact. The incision was made from inside the nostril away from the alar facial crease (Figs. 4-8). Cautery was usually not necessary and should be avoided as much as possible to avoid impaired wound healing and bad scarring. The wound was then closed by (2-3) simple interrupted 5/0 Prolene sutures. The same procedure was repeated on the other side.

\section{Postoperative care:}

Post-operative care and follow-up were the same as any rhinoplasty procedure.

The marginal, trans-columellar and alar reduction incisions are cleaned with normal saline and cotton swabs once a day, followed by application of topical antibiotic cream to keep the incisions moist and infection free and to reduce crusting.

Follow-up visits were after 24 hours, three days, one week (where the stitches were removed), then monthly for six months up to one year. 

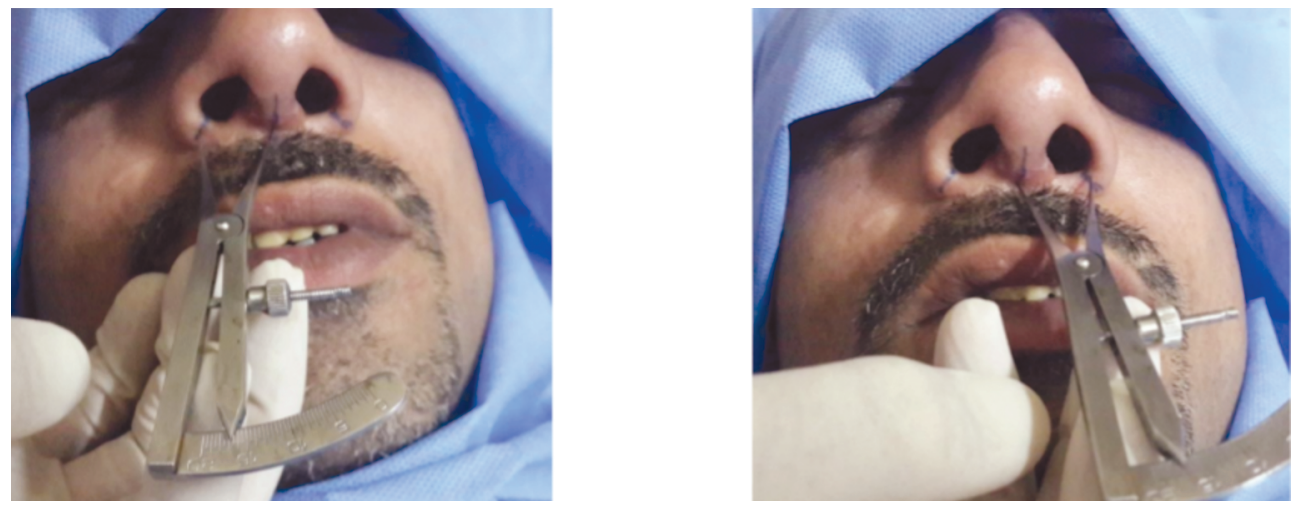

Fig. (4): A caliper is used to detect any asymmetry in the marking of the lateral limb of the triangular wedge.
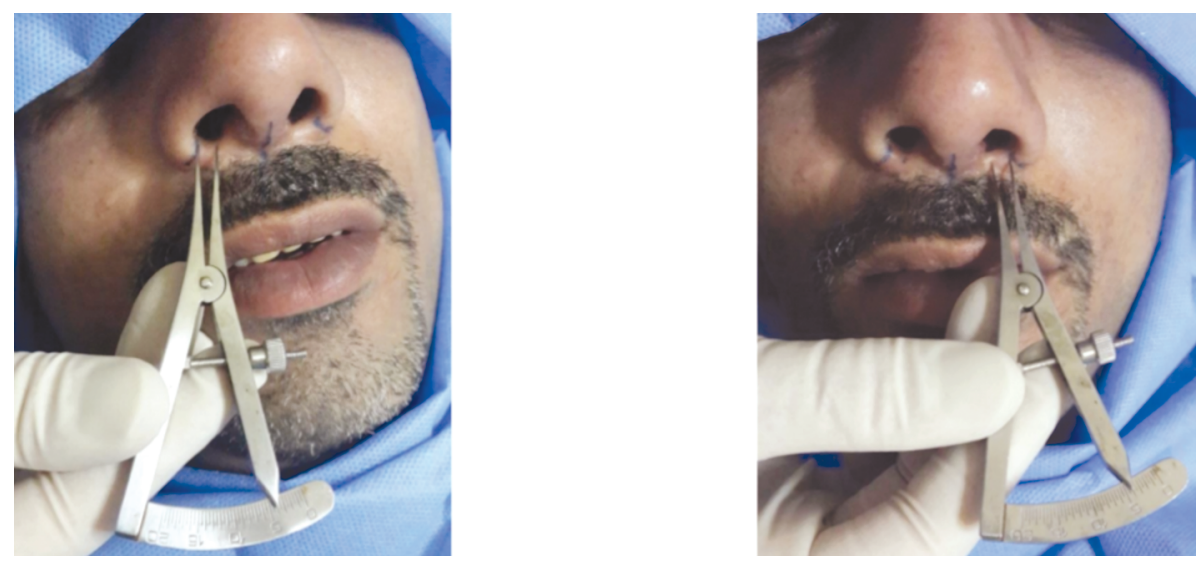

Fig. (5): The desired amount of narrowing of the outer diameter of the ala is determined. Caliper is used to sharply stab the skin from the lateral limb to desired sill crease to determine the medial limb of the triangular wedge to be excised.

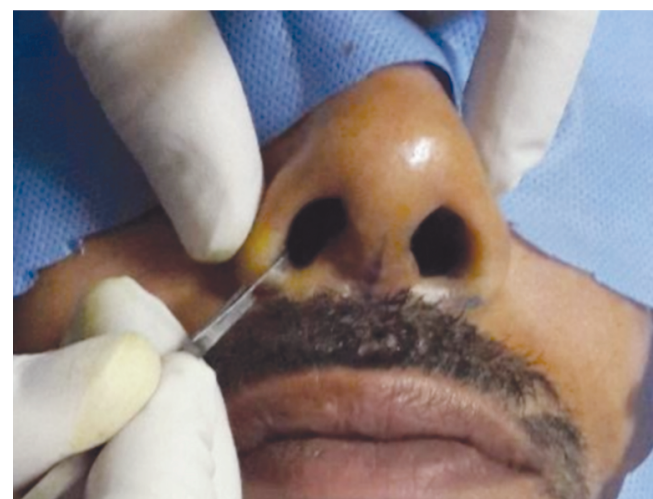

Fig. (6): Lateral and then medial sill incisions are made with a no. 15 blade.

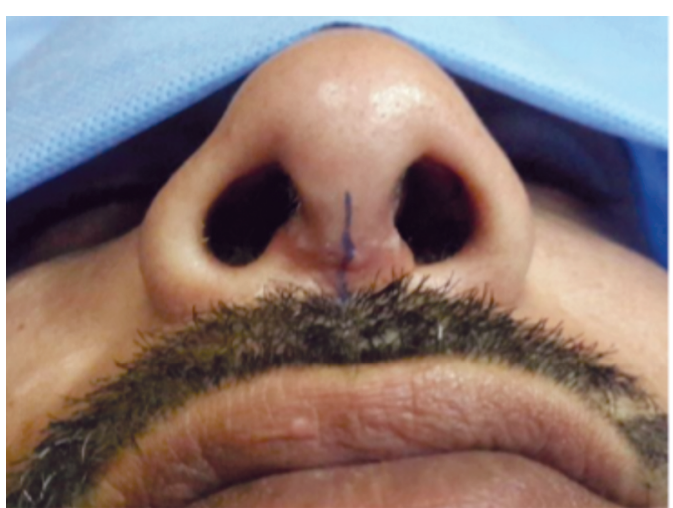

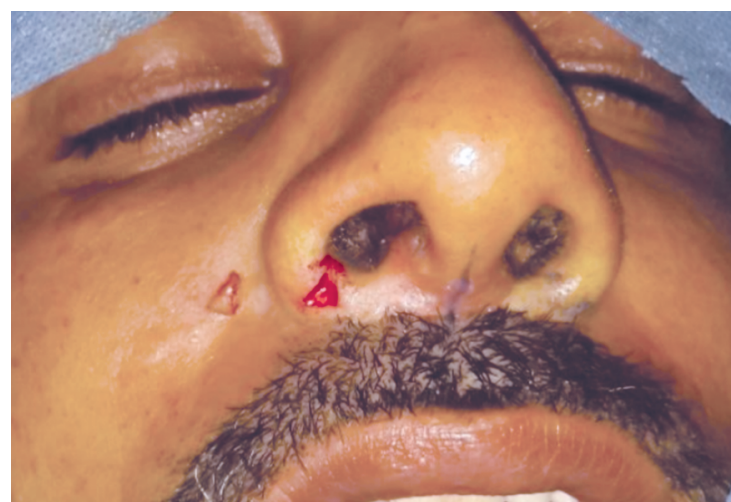

Fig. (7): The triangular wedge excision of skin, ensuring that the deep muscular layer remains intact.

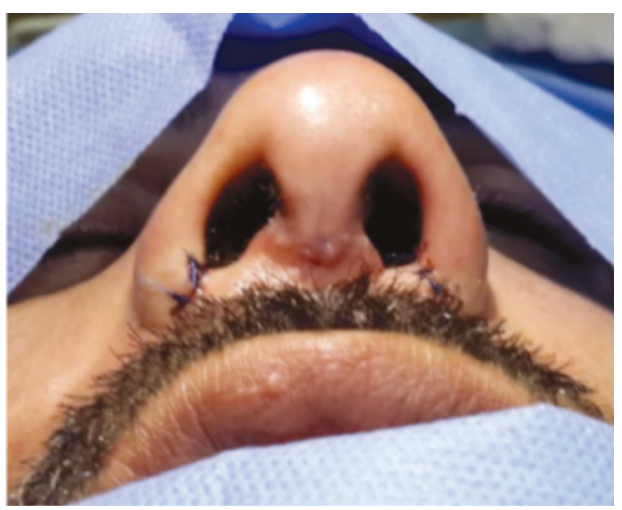

Fig. (8): Pre- and Post- operative of alar base reduction. 


\section{RESULTS}

In this prospective study 25 patients were included. Their ages ranged from 20 years to 37 years with a mean age of 28 . The female to male ratio was 3.2 to 1 , females comprising $76 \% .19$ cases were primary rhinoplasty (76\%). (Fig. 9).

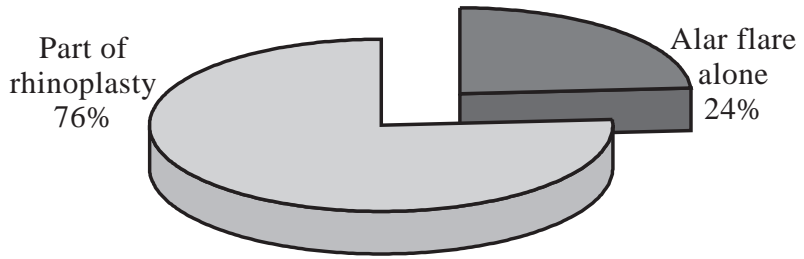

Fig. (9): Surgery percentage of alar flare alone vs being part of rhinoplasty.

\section{Alar flare:}

Inter-alar distance ranged from $(35-43 \mathrm{~mm})$ before surgery to become between $(31-37 \mathrm{~mm})$ after the surgery, with significant improvement ( $p$-value: $<0.001$ ). (Table 1).

Table (1): Alar flare before and after surgery.

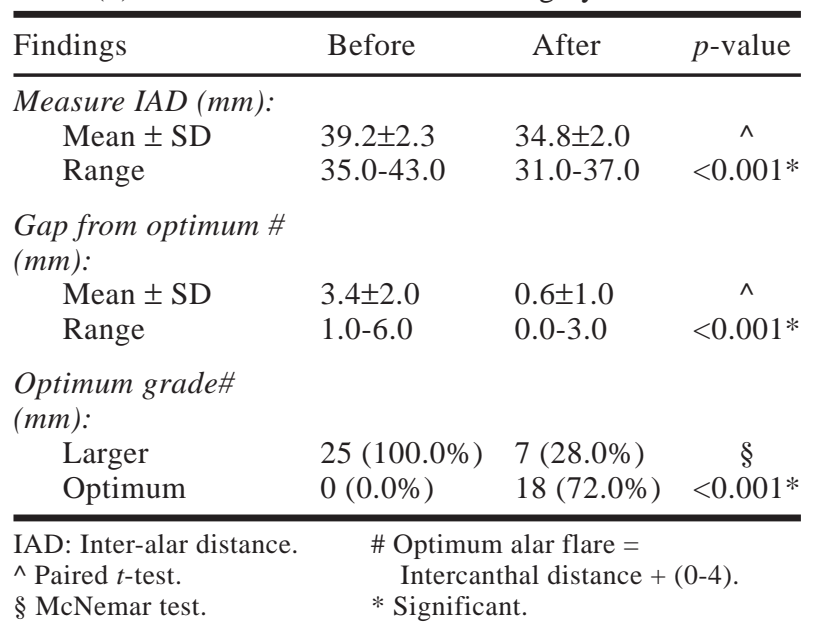

Prior to surgery all twenty-five patients complained of alar flaring (larger grade than the optimum). After surgery 18 patients $(72.0 \%)$ were completely corrected, and only 7 patients $(28.0 \%)$ were partially corrected. (Fig. 10).

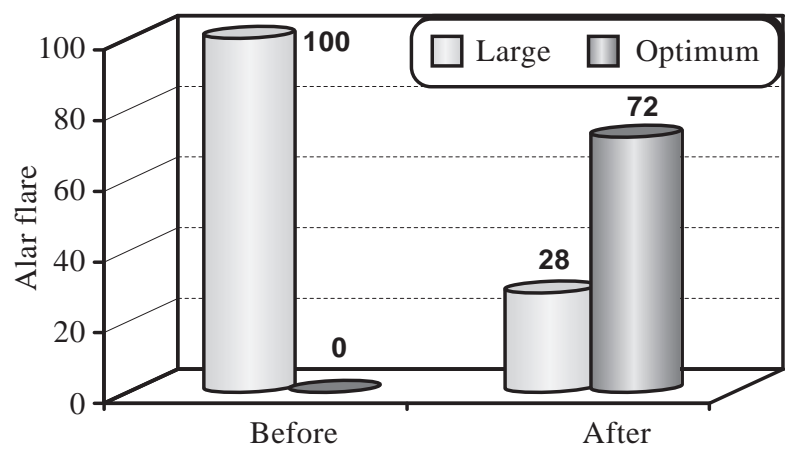

Fig. (10): Alar flare.
Gap from the optimum ranged between (1$6 \mathrm{~mm})$ before surgery to become between $(0-3 \mathrm{~mm})$ after the surgery, with significant improvement ( $p$ value: <0.001). Alar flare after surgery became significantly closer to optimum.

\section{Alar base:}

Alar base measurements ranged from (32$40 \mathrm{~mm}$ ) before surgery to become between (30$36 \mathrm{~mm}$ ) after the surgery.

Gap from the optimum ranged from (1-8mm) before surgery to become between ( -1 to $6 \mathrm{~mm}$ ) after surgery. (The optimum alar base is equal to the intercanthal distance).

Prior to surgery all twenty-five patients had wide alar base (larger grade than the optimum). After surgery 3 patients (12.0\%) had decreased alar base (by $1-2 \mathrm{~mm}$ ), to be equal to the intercanthal distance (optimum). One patient $(4.0 \%)$ had decreased alar base to be less than the intercanthal distance (lower than optimum). 21 patients (84.0\%) still had a wider alar base than the intercanthal distance (wider than optimum), but to a lesser degree than before surgery. (Table 2) (Fig. 11).

Table (2): Alar base before and after surgery.

\begin{tabular}{lllc}
\hline Findings & Before & After & $p$-value \\
\hline $\begin{array}{l}\text { Measure (mm): } \\
\quad \text { Mean } \pm \text { SD }\end{array}$ & $36.3 \pm 2.4$ & $33.8 \pm 2.1$ & $\wedge$ \\
$\quad$ Range & $32.0-40.0$ & $30.0-36.0$ & $<0.001^{*}$ \\
$\begin{array}{l}\text { Gap from optimum \# } \\
\text { (mm): }\end{array}$ & & \\
$\quad$ Mean \pm SD & $4.5 \pm 2.1$ & $2.1 \pm 2.0$ & $\wedge$ \\
$\quad$ Range & $1.0-8.0$ & $-1.0-6.0$ & $<0.001^{*}$ \\
Optimum grade \#: & & & \\
$\quad$ Larger & $25(100.0 \%)$ & $21(84.0 \%)$ & $\S$ \\
$\quad \begin{array}{l}\text { Optimum } \\
\text { Lower }\end{array}$ & $0(0.0 \%)$ & $3(12.0 \%)$ & $<0.001^{*}$ \\
\hline
\end{tabular}

\# Optimum alar base = Intercanthal distance

$\wedge$ Paired $t$-test. $\S \mathrm{McNemar}$ test. $\quad *$ Significant.

Negative values indicate lower than optimum cut off.

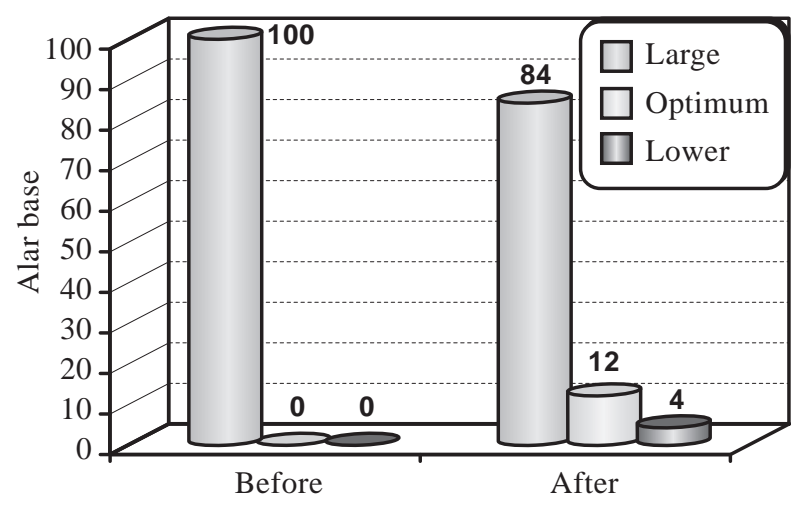

Fig. (11): Alar base. 


\section{Symmetry:}

Before surgery; 17 patients (68\%) had symmetrical nostrils, and after surgery; 15 patients (60\%) preserved their symmetrical nostrils, while only 2 patients $(8 \%)$ developed asymmetrical nostrils as a morbidity.

Prior to surgery; 8 patients (32\%) had asymmetrical nostrils, and after surgery; 4 patients $(16 \%)$ showed improvement with symmetrical nostrils, while 4 patients (16\%) still had residual asymmetry. (Table 3) (Fig. 12).

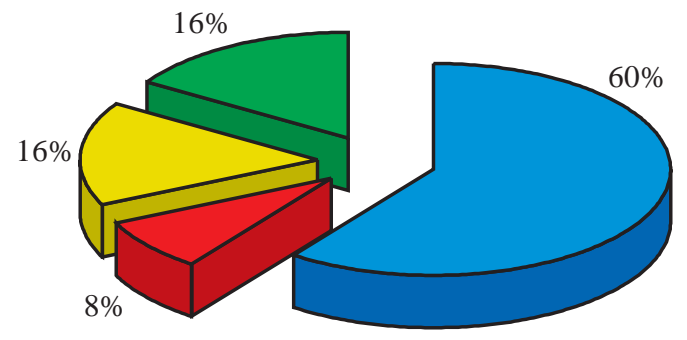

$92 \%$ of patients were satisfied with their postoperative results. Patient satisfaction was based on the improvement of nostrils shape and concealed scars.

\section{Complications:}

No notable complications, including infection, functional disturbances, hematomas, necrosis or deformity were noted during our study. There were

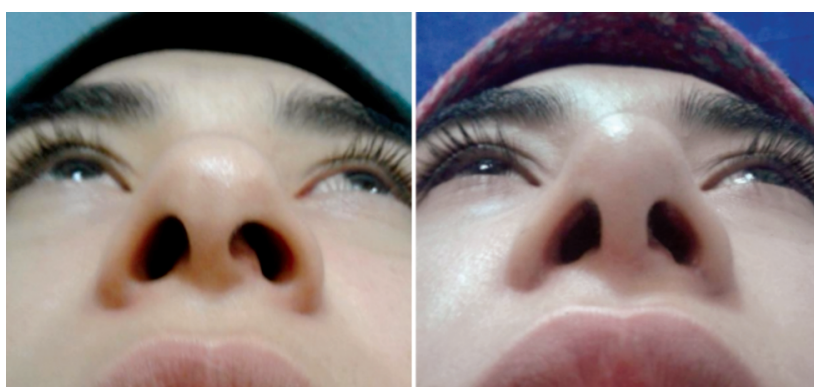

Fig. (13): Case 1 basal view (pre and postop.).

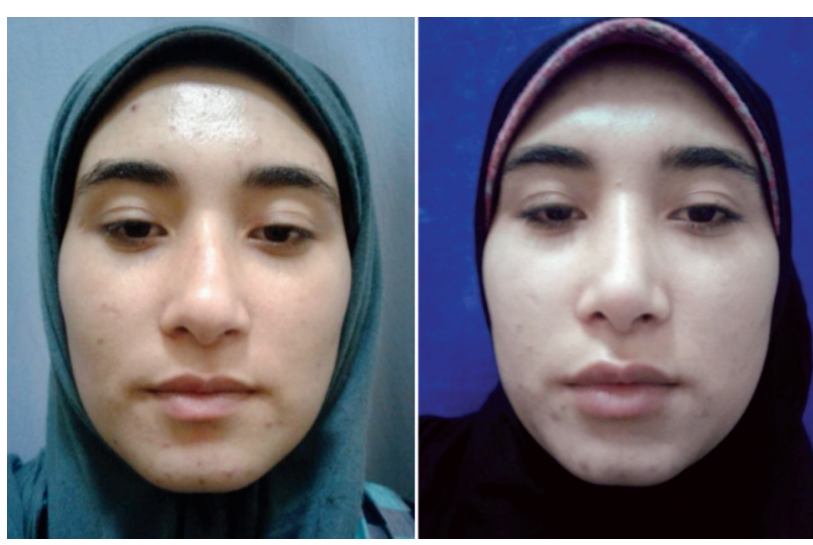

Fig. (14): Case 1 frontal view (pre and postop.).
Table (3): Nostril symmetry changes after surgery.

\begin{tabular}{llll}
\hline Before & \multicolumn{1}{c}{ After } & $\mathrm{N}$ & $\%$ \\
\hline Symmetrical nostrils & Symmetrical & 15 & 60.0 \\
$17(68 \%)$ & Asymmetrical & 2 & 8.0 \\
Asymmetrical nostrils & Symmetrical & 4 & 16.0 \\
$8(32 \%)$ & Asymmetrical & 4 & 16.0 \\
\hline
\end{tabular}

Total $=25$

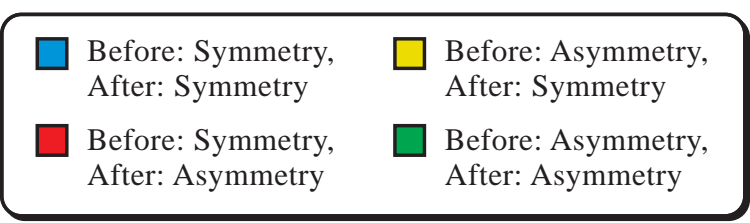

Fig. (12): Symmetry pre and postoperatively.

no healing problems or bad scarring in all patients during the follow-up period.

\section{Cases photography:}

- Case 1 (Fig. 13) (Fig. 14).

- Case 2 (Fig. 15) (Fig. 16).

- Case 8 (Fig. 17) (Fig. 18).

- Case 9 (Fig. 19) (Fig. 20).

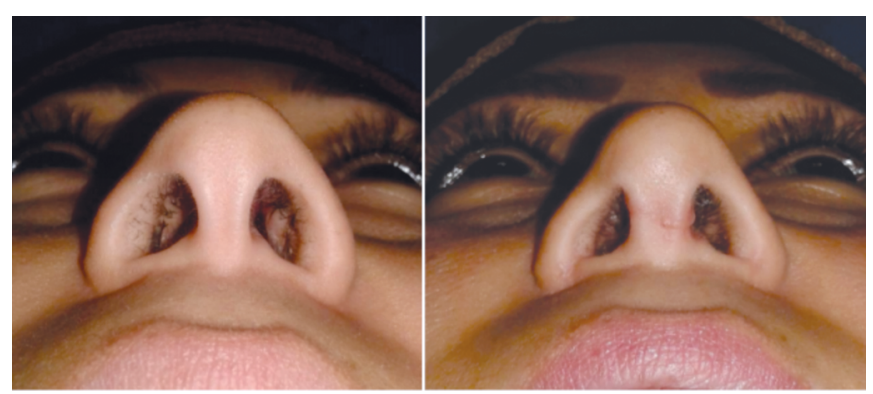

Fig. (15): Case 2 basal view (pre and postop.).
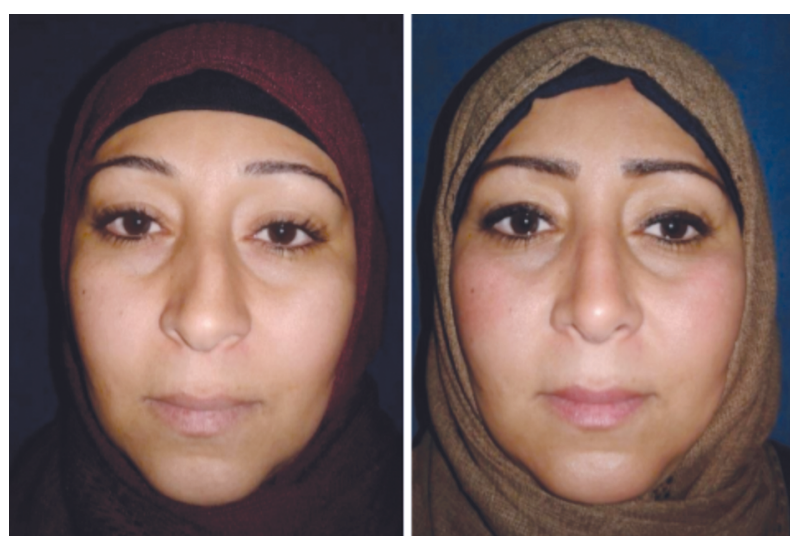

Fig. (16): Case 2 frontal view (pre and postop.). 

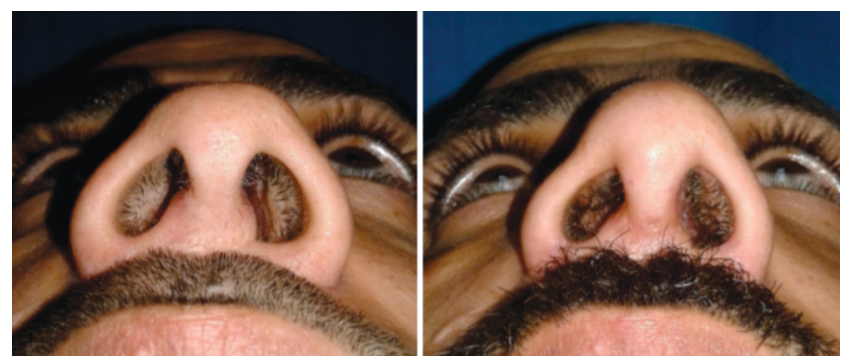

Fig. (17): Case 8 basal view (pre and postop.).
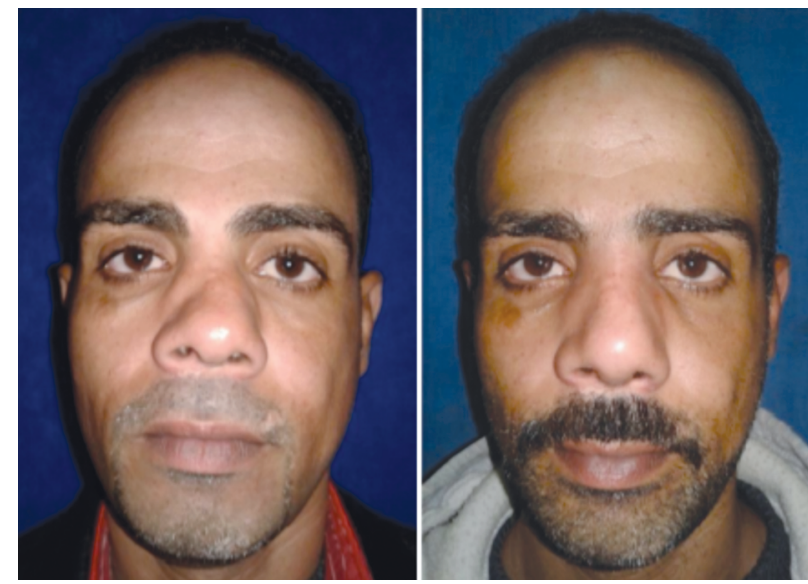

Fig. (18): Case 8 frontal view (pre and postop.).

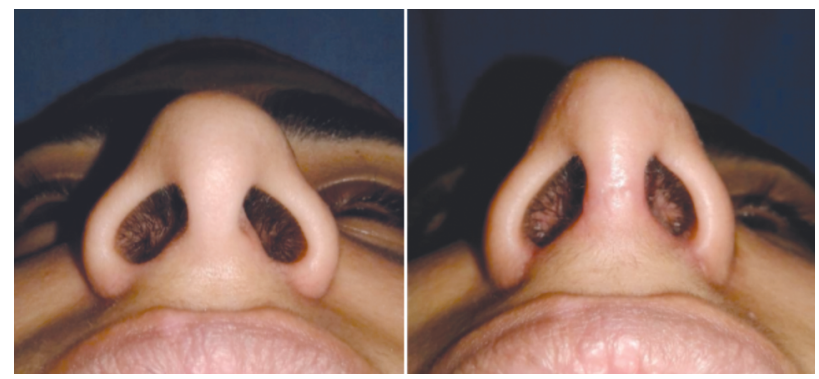

Fig. (19): Case 9 basal view (pre and postop.).
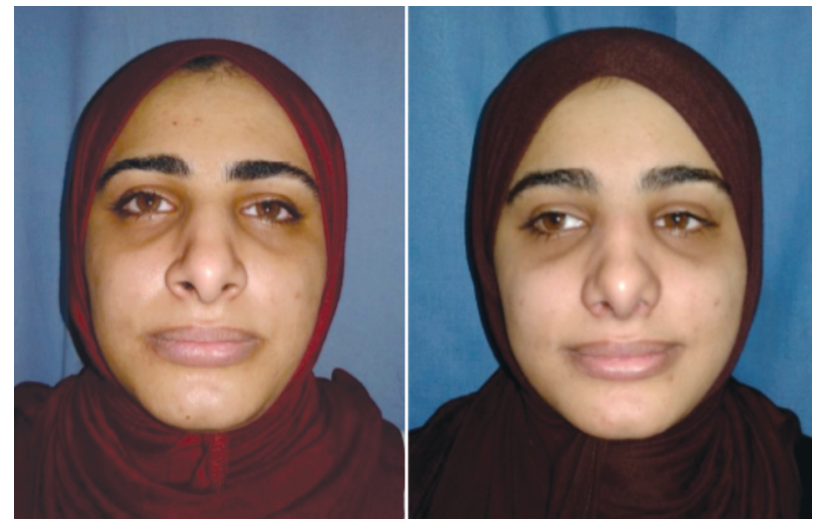

Fig. (20): Case 9 frontal view (pre and postop.).

\section{DISCUSSION}

Many techniques are available for alar base flare correction. These include sill excision, alar rim excision, cinching sutures, VY advancement or alar flap advancement techniques. The combination of sill and alar excision can used when alar lobule and vestibular floor parts are targeted [12].

In this study, we analyzed the surgical outcome of internal alar resection technique. The incision is made from internal aspect of the nostril, through a small triangular wedge excision from the medial wall of alar base and the nasal sill, away from alar facial crease. This technique combined alar wedge resection (to decrease the alar flare) with nostril sill resection (to approximate the alae) with the aim to decrease the alar flare and the width of the nasal base.

Optimum alar flare equals the intercanthal distance, and should not extend by more than 2 $\mathrm{mm}$ beyond the alar crease on either side. The alar flare is only considered if it was wider than the intercanthal distance by more than $4 \mathrm{~mm}$.

All 25 patients included in this study complained of alar flaring. 18 patients $(72 \%)$ were completely corrected according to the optimum aesthetic measures. Seven patients $(28 \%)$ showed improvement postoperatively, but still complained of minimal flaring.

The current study showed that the internal alar resection significantly decreases the alar flare; as the inter-alar distance ranged between $(35-43 \mathrm{~mm})$, Mean \pm SD (39.2 \pm 2.3$)$ before surgery and became between (31-37mm) Mean \pm SD (34.8 \pm 2.0$)$ after the surgery, with significant improvement ( $p$-value: $<0.001)$.

In the current study, six patients suffered alar flaring with wide nostrils alone; despite having a history of a previous rhinoplasty. They underwent alar base reduction under local anesthesia for correction of the flare and nostril shape, without any tip or septal surgery. The six patients improved and had symmetrical pear-shaped nostrils.

Many surgical techniques were employed for reduction of both the alar flare and the width of the nasal base. Each technique has its merits and drawbacks. As an example, cinching sutures may have the merit of avoiding external incisions but carry the disadvantage of leading to undue rounding of the alae due to crowding of the nasal floor.

This is opposed to our described technique, which addresses each sill separately, and is therefore useful even in cases of asymmetry. Consequently, the long-term effect of the cinching suture technique is considered doubtful [13]. 
Another important limitation of the cinching sutures is that it has a significantly high recurrence rate [14].

The main advantage of the VY-advancement technique is the reduction of wide inter-alar distance that cannot be resolved with wedge or sill excision alone. Yet, unlike our technique, the V-Y method results in an external, potentially visible scar [15].

In 2011 Ismail combined alar flap advancement technique to alar base excision. This technique has the merit of accuracy and nasal alar symmetry, but microdermabrasion is a must and has to be used as an adjuvant therapy to remove apparent scars [12].

Foda (2007), suggested that the combined alar base excision technique is effective for narrowing the alar base and reducing alar flare. The scar lies within the natural alar-facial crease and continues in the groove between the sill and upper lip. Although this scar is mostly inconspicuous, yet, an external scar still exists [5].

Many surgeons hesitate to perform alar base excision, in spite of its gratifying results, for fear of obvious scarring and unnatural outcome. Complications of alar soft tissue excision can include notching, apparent scars, asymmetry and nostril deformity [13]. The pull of the facial musculature may have the effect of widening the scar when the incision is placed in the crease [16].

However, in our patients, lobule notching and scarring were not observed, as the incision is made from inside the nostril, away from alar facial crease in order to avoid the pulling effect of facial musculature. Incision-related morbidity is lower with this technique than other techniques, which is suggested to be the most appropriate technique for narrowing inter-alar distance.

Bafaqeeh and Al-Quattan highlighted the risk ofpotential devascularization of the nasal flap with open rhinoplasty when combined with alar base resections [16,17].

Tip defattening of the nasal tip, especially when combined with open rhinoplasty and alar reduction, is considered hazardous to the tip vascularity as it violates the nasal tip plexus [17].

In the current study, the vascularization of the nasal tip is preserved by using the internal alar resection. This was achieved by limiting the incision inside the nostril and away from the lateral nasal artery.
It is of great importance to respect the basal view aesthetics and alar rim natural curve. Our described technique doesn't violate the natural curve or morphology, thus preventing any unnatural appearance of the ala or "Q" deformity, which may result from excisions of lateral alar wall [6].

Damage of "natural" nasal features as the alarfacial crease or disruption of the alar rim curvature can lead to unnatural and unwanted results [14]. It is of utmost importance to preserve the alar rim curvature when planning alar base reduction excision [18].

In the internal alar resection, the incision is made from inside the nostril; a small triangular wedge excision from the medial wall of alar base and the nasal sill. Thus, avoiding the main cause of obliteration of the natural alar-facial crease, which allows for maximal preservation of the natural curvature of the alar rim.

Symmetry is an esthetic key feature, and should be aimed at in all rhinoplasty or flare correction procedures. In alar base reduction, measuring before removal of tissue and careful reapproximation of skin edges can minimize asymmetry. Exact symmetry may not always be possible [19].

The technique described to minimize postoperative asymmetry is the "Calibrated Weir Operation" as described by Cinelli. A midcolumellar line is drawn from the nasolabial angle to the center of the superior vermillion border. An additional perpendicular line bisects this line and the columella. From the intersection of the 2 lines, one may measure the distance to the medial and lateral borders of a nasal base excision [18] (Fig. 4).

In this study, there is a significant increase in the frequency of nostril symmetry from 17 patients $(68 \%)$ to $19(76 \%)$, with significant improvement $(p<.001)$, due to using a similar technique prescribed by Kridel \& Castellano. They used to measure the distance from the mid-columellar point to the alar crease, on each side, to detect any asymmetry in nasal sill width which may entail different excisions to assure symmetry of nasal sill shape [19].

During our study we noticed that the main complication related to alar base excision was inadequate alar flare and nostrils asymmetry improvement. We had 2 patients $(8 \%)$ developed asymmetrical nostrils. Nevertheless, it is imperative to consult patients preoperatively concerning preexisting asymmetry, which cannot be fully corrected [6]. 
The combination of alar and sill excision to tip surgery can disturb the nostrils aesthetics-symmetry and shape. Therefore, when nostrils shape and symmetry are targeted, it is significant to have an integrated plan of tip surgery, reconstruction of the septum and alar base surgery, since perfect results cannot be achieved with alar excision alone [12].

Alar base narrowing was performed as the final step in rhinoplasty. It is better to judge the amount needed for alar base narrowing after closure of incisions and osteotomy are done, as it can be affected by nasal tip narrowing or tip projection change.

The majority of our patients were satisfied with their results (92\%). During follow-up visits questionnaires were done, with the aim of evaluating the important criteria that patients assess their results accordingly. Patient satisfaction was directly linked to hidden scars and to the improvement of nostril shape.

\section{Conclusion:}

Internal alar resection effectively reduced the alar flare, and improve the nostril shape and width. This technique was also useful to retain symmetry of nostril shape, hide the scars internally in the nose, avoid the injury of lateral nasal artery, preserve the natural curvature of alar rim and avoid detachment of alar foot plate. This technique can effectually be applied to all patients with alar flare reduction.

Declaration of conflict of interests: Authors have nothing to disclose.

Funding: This research did not receive any funding from any agency in the public, commercial, or not-for-profit sector.

All authors have made substantial contributions to all of the following: (1) Study idea, concept, technical, surgical contribution and study analysis (2) Article drafting and revision; (3) Final approval of the version to be submitted.

The manuscript, including related data, figures, and tables, has not been previously published and is not under consideration elsewhere.

Consent: Patients provided verbal and written consent for the procedure and participation in the study.

\section{REFERENCES}

1- Dickie, Sara R., Peter A., Adamson and Jeremy P. Warner: "Alar Base Reduction". Advanced Aesthetic Rhinoplasty. Springer, Berlin, Heidelberg, 425-434, 2013.

2- Adamson, Peter A., et al.: "Revision rhinoplasty: Panel discussion, controversies, and techniques". Facial Plastic Surgery Clinics, 22.1: 57-96, 2014.

3- Numa W. and C.M. Johnson Jr.: "Surgical anatomy and physiology of the nose". Master Techniques in Rhinoplasty. $20111^{\text {st }}$ ed Saunders Elsevier, 21-30, 2011.

4- Hudise, Jibril Y., et al.: "Evaluation of Scar Outcome After Alar Base Reduction Using Different Surgical Approaches". Journal of Oral and Maxillofacial Surgery, 2020.

5- Foda, Hossam M.T.: "Nasal base narrowing: The combined alar base excision technique". Archives of Facial Plastic Surgery, 9.1: 30-34, 2007.

6- Lima, Leila Freire Rego, Helena Hotz Arroyo and José Roberto Parisi Jurado: "Update in alar base reduction in rhinoplasty". Current Opinion in Otolaryngology \& Head and Neck Surgery, 24.4: 316-321, 2016.

7- Hamilton III and Grant S.: "YV alar base reduction". Ear, Nose \& Throat Journal, 93.3: 98-102, 2014.

8- Peng, Grace Lee and Paul S. Nassif: "Rhinoplasty in the African American patient: Anatomic considerations and technical pearls." Clinics in Plastic Surgery, 43.1: 255$264,2016$.

9- Harrar, Harpal, Simon Myers and Ali M. Ghanem: "Art or science? An evidence-based approach to human facial beauty a quantitative analysis towards an informed clinical aesthetic practice". Aesthetic Plastic Surgery, 42.1: 137$146,2018$.

10- Rohrich, Rod J., et al.: "Managing alar flare in rhinoplasty". Plastic and Reconstructive Surgery, 140.5: 910-919, 2017.

11- Casanueva, Fernando and Deniz Gerecci: "Objective comparison of nasal tip contours in the basal view between dome binding and hemitransdomal tip suture techniques in rhinoplasty". JAMA Facial Plastic Surgery, 18.6: 481487, 2016.

12- Ismail, Ahmed Soliman: "Nasal base narrowing: the alar flap advancement technique". Otolaryngology Head and Neck Surgery, 144.1: 48-52, 2011.

13- Kim, Ji Heui, Joon Pyo Park and Yong Ju Jang: "Aesthetic outcomes of alar base resection in Asian patients undergoing rhinoplasty". JAMA Facial Plastic Surgery, 18.6: 462-466, 2016.

14- Foda H.M.: "Nasal base reduction". J. Asthet. Chir., 7: 8$15,2014$.

15- Kumar S., M. Aslam and M. Yaseen: "Alar base resectionmedial flap technique". Indian Journal of Otolaryngology and Head and Neck Surgery, 58.4: 347-348, 2006.

16- Stucker, Fred J., Timothy Lian and Kenneth Sanders: "African American rhinoplasty". Facial Plastic Surgery Clinics, 13.1: 65-72, 2005. 
17- Bafaqeeh, Sameer A. and Mohammad M. Al-Qattan: "Simultaneous open rhinoplasty and alar base excision: Is there a problem with the blood supply of the nasal tip and columellar skin?". Plastic and Reconstructive Surgery, 105.1: 344-349, 2000.

18- Scuderi, Nicolò and Bryant A. Toth, eds.: International Textbook of Aesthetic Surgery. Springer, 2016
19- Kridel, Russell W.H. and Richard D. Castellano: "A simplified approach to alar base reduction: A review of 124 patients over 20 years". Archives of Facial Plastic Surgery, 7.2: 81-93, 2005.

20- Uzun, Ahmet and Fikri Ozdemir: "Morphometric analysis of nasal shapes and angles in young adults". Brazilian Journal of Otorhinolaryngology, 80.5: 397-402, 2014. 